\title{
Spectral Observations of Filament Activation
}

\author{
G. Mashnich \\ Institute of Solar-Terrestrial Physics, SB RAS, P. O. Box 4026, Irkutsk, 664033 Russia \\ email: mashnich@iszf.irk.ru
}

\begin{abstract}
Studies of solar filament (prominence) activation and eruption are often based on measurements of intensity fluctuations in various solar emission bands and rarely on Doppler velocity measurements. The goal of this paper is to analyze the process of quiescent filament activation, using spectral data, and its associated events in solar UV band. Motions have been examined in a small southern fragment of a quiescent, extended filament in the northern hemisphere prior to and during its activation on June 14 2012. A part of the fragment disappeared after the filament activation.
\end{abstract}

\section{Observations and method of data processing}

The quiescent filament was observed by Sayan Solar Observatory's horizontal solar telescope prior to and during the activation on June 142012 (E11N27). A series of spectra in the $H \beta$ line region (including $\lambda 486.1 \mathrm{~nm}$ and Fe I $\lambda 489.7 \mathrm{~nm}$ in the chromosphere and photosphere respectively) was registered from 01:43 UT to 04:18 UT with a cadence of $10 \mathrm{~s}$ between the consecutive spectra. The mirror spectrograph's slit and an auxiliary device with an $H \alpha$-filter provide $H \alpha$-filtergrams together with an actual slit position against the filament body. The spectral slit position covered the region of filament bifurcation. The analysis of dynamics of motions in the filament also involves simultaneous events in SDO/AIA, SDO/HMI magnetograms and BBSO $H \alpha$-images. The SDO magnetograms show a complex configuration of weak polarities in the photospheric magnetic field under the region of the filament bifurcation. Velocities perpendicular to the line of sight are measured by the method of lowest intensity of $H \beta$ line (Mashnich et al. (2012)) and the Doppler velocity by the bisector method. Wavelet analysis for the prior to activation time interval (between 01:43UT to 03:05 UT) carried out to investigate time behaviors of the filament and the photosphere under filament Doppler velocity oscillations. The sequence of images SDO/AIA images in He II $(30.4 \mathrm{~nm})$ is processed by subtracting the first image prior to the filament activation from each subsequent image.

\section{Results and discussions}

Short-period (about 5 minutes) oscillations occurred across the whole width of the filament 25 minutes before the filament activation. Such oscillations are typical only for fine structures which are well-resolved at filament edges. For a given time period the profile of the velocity distribution along the slit in the photosphere was shown to reoccur in the filament with a delay of 260-280 sec. Assuming the filament height to be at 50 $\mathrm{Mm}$ above the photosphere, we estimated the travel velocity of the disturbance from the photosphere to the filament to be between $110 \mathrm{~km} / \mathrm{s}$ and $180 \mathrm{~km} / \mathrm{s}$.

The filament activation is illustrated by the spectra (Fig. 1). In the first phase, the core filament structure rose; after that time, the edges of the filament channel were being traced by bright points in He II $(30.4 \mathrm{~nm})$. With the increasing rising velocity, the filament structure broke off, a bright region flared up in He II $(30.4 \mathrm{~nm})$, and, after the 

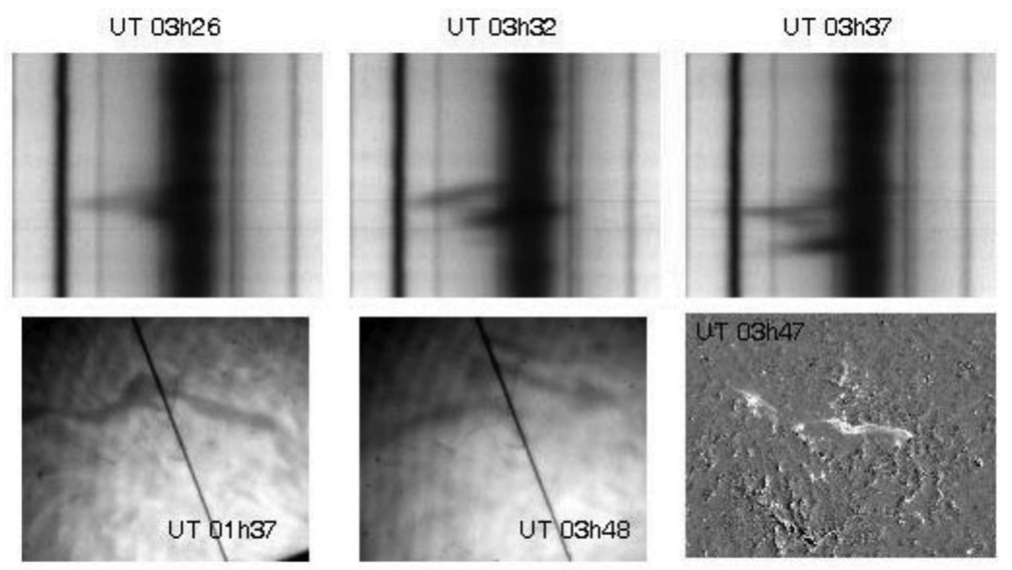

Figure 1. Top panel shows the sequence of spectra in the $H \beta$ line during the filament activation. The bottom panel shows the filament position on the mirror spectrograph's slit (black line) at the beginning of observations (left) and during the filament activation (middle) in the blue wing of the $H \alpha$ line. The difference image in He II $(30.4 \mathrm{~nm})$ during the filament activation, is on the right. Each image is labelled with the universal time (UT) at which it was taken.

maximum of filament rise velocity $(75-95 \mathrm{~km} / \mathrm{s})$, bright arches began to spread from this point to opposite sides of the filament channel (Fig. 1). The bright arches were visible in other bands of SDO/AIA. This process repeated after about 50 minutes. The $H \alpha-$ filament split into fragments for a short time during the filament activation and some of them disappeared (Fig. 1). The reason for the filament destabilization and acceleration is still unclear. It should be noted that there is a time correlation between the activation of this filament located in the North hemisphere with flare events in AR 11504 located in the South hemisphere visible in He II $(30.4 \mathrm{~nm})$ and other bands of SDO/AIA. The activation of this filament can be compared with the dynamics observed in a large quiescent polar crown filament observed with STEREO (Gosain et al. (2009)). Gosain et al. (2009) measured apparent velocities of the same order. We are measuring plasma Dopplershifts.

I am thankful to Dr. B. Schmieder for useful comments and discussion of results. I would also like to thank Mashnich V.I. for his help in data processing. This study was supported by the Programme of the Basic Fundamental Research of SB RAS No. 16.1.

\section{References}

Gosain, S., Schmieder, B., Venkatakrishnan, R. Chandra, \& Artzner, G. 2009, Solar Phys, 259, 13

Mashnich, G. P., Bashkirtsev, V. S., \& Khlystova, A. I. 2012, Astronomy Reports, v. 56, 3, 241 